\title{
Iris Recognition Methods - Survey
}

\author{
$S$ V Sheela \\ B M S College of \\ Engineering \\ Bangalore, India.
}

\author{
P A Vijaya \\ Malnad College of Engineering \\ Hassan, India
}

\begin{abstract}
The premise is that a biometric is a measurable physical characteristic which are reliable than passwords. Iris biometry is used to recognize an individual in a natural and intuitive way. Secure communications and mobile commerce are some of the application areas. Iris based security applications thrive on infrared cameras and video cameras for logins and transaction authentications. Accuracy, algorithm speed and template size are attributes that are important for large-scale identity programs and national database applications. In this paper, different iris recognition methods which aid an appropriate outlook for future work to build integrated classifier on latest input devices for excellent business transactions are discussed. Benchmark databases, products are also discussed. Since the area is currently one of the most on the go and the bulk of research is very large, this survey covers some of the significant methods.
\end{abstract}

\section{Keywords}

Iris Recognition, Phase based method, Texture-analysis, Zero crossing, Local intensity variations, Independent Component Analysis, Continuous Dynamic Programming.

\section{INTRODUCTION}

Biometrics deals with automated methods of recognizing a person based on physiological characteristics such as face, fingerprints, hand geometry, iris, retinal, and vein. Biometric authentication technique based on iris patterns is suitable for high level security systems. Iris is the annular ring between the pupil and the sclera of the eye. The structure of iris is fixed from about one year in age and remains constant over time. It exhibits long-term stability and infrequent re-enrolment requirements. The variations in the gray level intensity values distinguish two individuals. The difference exists between identical twins and even between left and right eye of the same person. As the technology is iris pattern-dependent, not sight dependent, it can be used by blind people. The iris is highly protected, noninvasive and ideal for handling applications requiring management of large user groups, like voter ID management. The iris recognition techniques potentially prevent unauthorized access to ATMs, cellular phones, desktop PCs, workstations, buildings and computer networks. The accuracy of iris recognition systems is proven to be much higher compared to other types of biometric systems like fingerprint, handprint and voiceprint.

In the year 1885, a French ophthalmologist, Alphonse Bertillon first proposed iris pattern as a basis for personal identification
[1]. In 1987, Flom and Safir [2] obtained an unimplemented concept of automated iris biometrics system. A report was published by Johnston [3] in 1992 without any experimental results. Iris based security systems capture iris patterns of individuals and match these patterns against the record in available databases. Even though significant progress has been made in iris recognition, handling noisy and degraded iris images require further investigation. The iris recognition algorithms need to be developed and tested in diverse environment and configurations. Research issues are based on iris localization, nonlinear normalization, occlusion, segmentation, liveness detection and large scale identification. It is required to achieve lowest false rejection rate and fastest composite time for template creation and matching.

A typical iris recognition system involves four main modules.

- The first module, image acquisition deals with capturing sequence of iris images from the subject using cameras and sensors. An image acquisition consists of illumination, position and physical capture system. The occlusion, lighting, number of pixels on the iris are factors that affect the image quality [4]. Many iris recognition systems require stern cooperation of the user for image acquisition. Ketchantang [5] proposed a method in which the entire sequence of images is acquired during enrolment and the best feasible images are selected, to increase flexibility. Enrolment aids to provide strong identity management.

- The second module, preprocessing involves various steps such as iris liveness detection, pupil and iris boundary detection, eyelid detection and removal and normalization. Iris liveness detection differentiates live subject from a photograph, a video playback, a glass eye or other artifacts. It is possible that biometric features are forged and illegally used. Several methods like Hough transformation, integrodifferential operator, gradient based edge detection are used to localize the portions of iris and the pupil from the eye image. The contours of upper and lower eyelids are fit using the parabolic arcs resulting the eyelid detection and removal. It is essential to map the extracted iris region to a normalized form. The iris localization methods are based on spring force, morphological operators, gradient, probability and moments. Iris localization method developed by Zhaofeng He [6] is based on spring force-driven iteration scheme using Hooke's law. The composition of forces from all points determines the centre and radius of pupil and iris. Morphological operators were applied by Mira and Mayer [7] to obtain iris boundaries. The inner boundary is detected 
by applying threshold, image opening and closing operators. The outer boundary is detected by applying threshold, closing and opening operators. The iris localization method by Guodong Guo [8] is based on intensity gradient and texture difference. The intensity gradient use integrodifferential operator. The Kullback-Leibler divergence is used to measure the distance between two probability distributions derived from the inner and outer zones. H. Proenca and L.A.Alexandre [9] proposed a moment-based texture segmentation algorithm, using second order geometric moments of the image as texture features. The clustering algorithms like self-organizing maps, $\mathrm{k}$ means and fuzzy k-means were used to segment the image to produce as output the clusters-labeled images. The experiments were conducted on UBIRIS database with accuracy of $98.02 \%$ and $97.88 \%$ for images captured in session 1 and session 2, respectively. The segmentation performance for 1214 good quality images and 663 noisy images was $98.02 \%$ and $97.88 \%$, respectively.

- The third module, feature extraction identifies the most prominent features for classification. Some of the features are $x-y$ coordinates, radius, shape and size of the pupil, intensity values, orientation of the pupil ellipse and ratio between average intensity of two pupils. The features are encoded to a format suitable for recognition.

- The fourth module, recognition achieves result by comparison of features with stored patterns [10]. The interclass and intra-class variability are used as metrics for pattern classification problems.

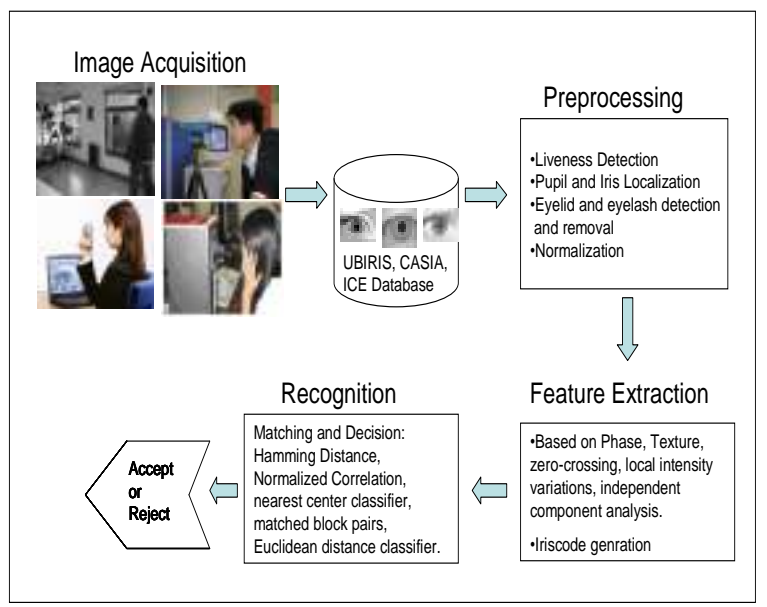

Figure 1. Iris Recognition System.

\section{IRIS IMAGE DATASETS}

The accuracy of the iris recognition system depends on the image quality of the iris images. Noisy and low quality images degrade the performance of the system. UBIRIS database is the publicly available database [9]. It consists of images with noise, with and without cooperation from subjects. The UBIRIS database has two versions with images collected in two distinct sessions corresponding to enrolment and recognition stages. The second version images were captured with more realistic noise factors on non-constrained conditions such as at-a-distance, on-the-move and visible wavelength. CASIA iris image database images are captured in two sessions [29]. CASIA-IrisV3 contains a total of 22,051 iris images from more than 700 subjects. It also consists of twins' iris image dataset. ND 2004-2005 database is the superset of Iris Challenge Evaluation (ICE) dataset, uses an Iridian iris imaging system for capturing the images [16]. The system provides voice feedback to guide the user to the correct position. The images are acquired in groups of three called as shot. For each shot, the system automatically selects the best image of the three and reports values of quality metrics and segmentation results for that image. For each person, the left eye and right eye are enrolled separately. The specifications of databases are listed in Table 1.

\section{IRIS RECOGNITION METHODS}

\subsection{Phase-based method}

The phase based method recognize iris patterns based on phase information. Phase information is independent of imaging contrast and illumination. J.Daugman [11, 12] designed and patented the first complete, commercially available phase-based iris recognition system in 1994 . The eye images with resolution of 80-130 pixels iris radius were captured with image focus assessment performed in real time. The pupil and iris boundary was found using integrodifferential operator given in Equation (1).

$\max _{\left(r, x_{0}, y_{0}\right)}\left|G_{\sigma}(r) * \frac{\partial}{\partial r} \int_{r, x_{0}, y_{0}} \frac{I(x, y)}{2 \pi r} d s\right|$

where $\mathrm{I}(\mathrm{x}, \mathrm{y})$ is the image in spatial coordinates, $\mathrm{r}$ is the radius, $(\mathrm{x} 0, \mathrm{y} 0)$ are centre coordinates, the symbol $*$ denotes convolution and $\mathrm{G} \sigma(\mathrm{r})$ is a Gaussian smoothing function of scale $\sigma$. . The centre coordinates and radius are estimated for both pupil and iris by determining the maximum partial derivative of the contour integral of the image along the circular arc. The eyelid boundaries are localized by changing the path of contour integration from circular to arcuate. The iris portion of the image $\mathrm{I}(\mathrm{x}, \mathrm{y})$ is normalized to the polar form by the mapping function $\mathrm{I}(\mathrm{x}(\mathrm{r}, \theta), \mathrm{y}(\mathrm{r}, \theta)) \rightarrow \mathrm{I}(\mathrm{r}, \theta)$ where $\mathrm{r}$ lies on the unit interval $[0,1]$ and $\theta$ is the angular quantity in the range $[0,2 \pi]$. The representation of iris texture is binary coded by quantizing the phase response of a texture filter using quadrature 2D Gabor wavelets into four levels. Each pixel in the normalised iris pattern corresponds to two bits of data in the iris template. A total of 2,048 bits are calculated for the template, and an equal number of masking bits are generated in order to mask out corrupted regions within the iris. This creates a compact 256byte template, which allows for storage and comparison of iris. 
The recognition in this method is the failure of a test of statistical independence involving degrees of freedom. Iriscodes are different for two different samples. The test was performed using boolean XOR operator applied to 2048 bit phase vectors to encode any two iris patterns, masked (ANDed) by both of their corresponding mask bit vectors. From the resultant bit vector and mask bit vectors, the dissimilarity measure between any two iris patterns is computed using Hamming Distance (HD) given in Equation (2).

Table 1: Database specifications

\begin{tabular}{|c|c|c|c|c|c|c|c|}
\hline Database & Research Lab & Version & $\begin{array}{l}\text { Camera used for } \\
\text { image } \\
\text { acquisition }\end{array}$ & Images & Subjects & Format & Resolution \\
\hline \multirow[t]{2}{*}{ UBIRIS } & \multirow{2}{*}{$\begin{array}{l}\text { SOCIA Lab. } \\
\text { Computing and Image } \\
\text { Analysis } \\
\text { Group, Department } \\
\text { Computer of } \\
\text { University of Beira Interior, } \\
\text { Portugal }\end{array}$} & V1 & $\begin{array}{l}\text { Nikon E5700 } \\
\text { camera }\end{array}$ & 1877 & 241 & jpeg & $400 \times 300$ \\
\hline & & $\mathrm{V} 2$ & Canon EOS 5D & 11,102 & 261 & jpeg & $800 \times 600$ \\
\hline \multirow{5}{*}{ CASIA } & \multirow{5}{*}{$\begin{array}{l}\text { Iris Recognition Research } \\
\text { Group, Center for } \\
\text { Biometrics and Security } \\
\text { Research, } \\
\text { Laboratory of Natioanal } \\
\text { Recognition, Institute of } \\
\text { Automation, Chinese } \\
\text { Academy of Sciences, } \\
\text { Beijing, China. }\end{array}$} & V1 & Self-developed & 756 & 108 & bmp & $320 \times 280$ \\
\hline & & V2 & Self-developed & 1200 & 60 & bmp & $640 \times 480$ \\
\hline & & V3-Interval & Self-developed & 2655 & 249 & jpeg & $320 \times 280$ \\
\hline & & V3-Lamp & OKI & 16213 & 411 & jpeg & $640 \times 480$ \\
\hline & & V3-Twins & OKI & 3183 & 200 & jpeg & $640 \times 480$ \\
\hline ND 2004-2005 & $\begin{array}{l}\text { Dept. of Computer Science } \\
\text { and Engineering, University } \\
\text { of Notre Dame, Notre } \\
\text { Dame, United States of } \\
\text { America }\end{array}$ & - & $\begin{array}{l}\text { Iridian LG } \\
\text { EOU2200 }\end{array}$ & 64,980 & 356 & tiff & $640 \times 480$ \\
\hline \multirow[t]{3}{*}{$\begin{array}{l}\text { University of } \\
\text { Bath Iris Image } \\
\text { Database }\end{array}$} & \multirow[t]{3}{*}{$\begin{array}{l}\text { University of Bath, Bath, } \\
\text { United Kingdom }\end{array}$} & Iris DB 400 & \multirow{3}{*}{$\begin{array}{l}\text { AD-100 } \\
\text { IrisGuard Dual- } \\
\text { Eye autofocus } \\
\text { camera }\end{array}$} & 8,000 & 200 & bmp & $1280 \times 960$ \\
\hline & & Iris DB 800 & & 16,000 & 400 & bmp & $1280 \times 960$ \\
\hline & & Iris DB 1600 & & 32,000 & 800 & bmp & $1280 \times 960$ \\
\hline UPOL & $\begin{array}{l}\text { Dept. Computer Science, } \\
\text { Palacky University, } \\
\text { Olomouc }\end{array}$ & - & $\begin{array}{l}\text { SONY DXC- } \\
\text { 950P 3CCD } \\
\text { camera }\end{array}$ & 384 & 64 & png & $576 \times 768$ \\
\hline \multirow[t]{2}{*}{ MMU } & \multirow{2}{*}{$\begin{array}{l}\text { Multimedia University, } \\
\text { Malaysia }\end{array}$} & MMU1 & $\begin{array}{l}\text { LG IrisAccess } \\
2200\end{array}$ & 450 & 100 & bmp & $320 \times 280$ \\
\hline & & MMU2 & $\begin{array}{l}\text { Panasonic BM- } \\
\text { ET100US } \\
\text { Authenticam }\end{array}$ & 995 & 100 & bmp & $320 \times 280$ \\
\hline
\end{tabular}

$H D=\frac{\|(\operatorname{code} A \otimes \operatorname{code} B) \cap \operatorname{mask} A \cap \operatorname{mas} k B\|}{\| \text { mask } A \cap \operatorname{mask} B \|}$

Where codeA, codeB are two phase code bit vectors and maskA, maskB are mask bit vectors. The HD is a fractional measure of dissimilarity with 0 representing a perfect match. A low normalized HD implies strong similarity of iris codes.

The work by Xianchao Qui [13] used 2D Gabor filters for localization. The filter response vectors were clustered using vector quantization algorithms like k-means. The experiments 
were conducted on CASIA-Biosecure iris database consisting of images captured from Asian and non-Asian race groups. The support vector machine was used for the two class ethnic classification. The methodology proposed by Martin-Roche [14] and Masek [15] operates in the same concept of Daugman for recognition. In Martin's method, the iris circumference parameters are obtained by maximising the average intensity differences of the five consecutive circumferences. In Masek's method, the segmentation was based on the Hough transform. The phase data from 1D Log-Gabor filters was extracted and quantised to four levels to encode the unique pattern of the iris into a bit-wise biometric template. Xiaomei Liu [16] reimplemented Masek's algorithm in C that was originally written in Matlab. Continuing the Daugman's method, Karen Hollingsworth [17] has developed a number of techniques for improving recognition rates. These techniques include fragile bit masking, signal-level fusion of iris images, and detecting local distortions in iris texture. The bits near the axes of the complex plane shifts the filter response from one quadrant to adjacent quadrant in presence of noise. In the fragile bit masking method, such bits called as the fragile bits are identified and masked to improve the accuracy. The signal-level fusion method uses image averaging of selected frames from a video clip of an iris. Local texture distortions occurs with contact lenses with a logo, poor-fit contacts and edges of hard contact lenses, segmentation inaccuracies and shadows on the iris. These are detected by analysing iris code matching results. The 20x240 normalized images were covered with 92 windows each of size $8 \times 20$. Fractional HD was computed for each window. The location of windows with highest fractional HD was identified and removed from further calculations. The effect of dilation was studied by collecting datasets of images with varying degrees of dilation. The data was divided into subsets with small pupils, medium pupils and large pupils. The subset of data with large pupils showed worst performance with EER at an order of magnitude greater compared to that of small pupil data set. The visibility in the iris area is reduced and greater part of iris is occluded by eyelids which provide less information for iriscode generation.

\subsection{Texture-analysis based method}

Wildes proposed iris recognition based on texture analysis [18, 19, 20]. High quality iris images was captured using silicon intensified target camera coupled with a standard frame grabber and resolution of 512x480 pixels. The limbus and pupil are modeled with circular contours which is extended to upper and lower eyelids with parabolic arcs. The particular contour parameter values $\mathrm{x}, \mathrm{y}$ and radius $\mathrm{r}$ are obtained by the voting of the edge points using Hough transformation. The largest number of edge points represents the contour of the iris. The Laplacian of Gaussian (LoG) is applied to the image at multiple scales and Laplacian pyramid is constructed. The LoG filter is given in Equation (3).

$$
-\frac{1}{\pi \sigma^{4}}\left(1-\frac{\rho^{2}}{2 \sigma^{2}}\right) e^{\rho^{2} / 2 \sigma^{2}}
$$

where $\sigma$ is the standard deviation of the Gaussian and $\rho$ is the radial distance of a point from the filter's centre. The matching is based on normalised correlation between the acquired and database images. Classification is performed using Fisher's linear discriminant function. The method for iris identification by Emine Krichen [21] use a hybrid method for iris segmentation, Hough transform for outer iris boundary and integrodifferential operator for inner iris boundary. The iris code was produced using wavelet packets. The whole image is analyzed at different resolutions. 832 wavelets with 4 scales are used to generate 1664 bits code. The iris database consisted of 700 images acquired with visible light. An improvement of $2 \%$ FAR and $11.5 \%$ FRR was obtained relative to Daugman method. It was observed that by considering colour information, overall improvement of $2 \%$ to $10 \%$ was obtained according to threshold value.

\subsection{Zero-Crosssing representation method}

The method developed by Boles [22] represents features of the iris at different resolution levels based on the wavelet transform zero-crossing. The algorithm is translation, rotation and scale invariant. The input images are processed to obtain a set of 1D signals and its zero crossing representation based on its dyadic wavelet transform. The wavelet function is the first derivative of the cubic spline. The centre and diameter of the iris is calculated from the edge-detected image. The virtual circles are constructed from the center and stored as circular buffers. The information extracted from any of the virtual circles is normalised to have same number of data points and a zero crossing representation is generated. The representation is periodic and independent from the starting point on iris virtual circles. These are stored in the database as iris signatures. The dissimilarity between the iris of the same eye images was smaller compared to the eye images of different eyes. The advantage of this function is that the amount of computation is reduced since the amount of zero crossings is less than the number of data points. But the drawback is that it requires the compared representations to have the same number of zero crossings at each resolution level.

\subsection{Approach based on intensity variations}

Iris recognition system developed by $\mathrm{Li} \mathrm{Ma}$ is characterized by local intensity variations [10]. The sharp variation points of iris patterns are recorded as features. In the iris localization phase, the centre coordinates of the pupil are estimated by image projections in horizontal and vertical directions. The exact parameters of the pupil and iris circles are calculated using Canny edge detection operator and Hough transform. The iris in cartesian coordinate system is projected into a doubly dimensionless pseudopolar coordinate system. The local spatial patterns in an iris consist of frequency and orientation information. Gabor filters are constructed to acquire frequency band in the spatial domain. Gabor functions are Gaussians modulated by circularly symmetric sinusoidal functions. The feature extraction begins by generating 1D intensity signals considering the information density in the angular direction. The 1D signal is represented using dyadic wavelet transform to obtain the feature vector. It decomposes the signal into detail components at different scales. The feature values are the mean and the average absolute deviation of the magnitude of each $8 \times 8$ block in the filtered image with the total number of blocks being 768. For dimensionality reduction, Fisher Linear Discriminant is used and for classification, nearest centre classifier is used. The similarity between the pair of feature vectors is calculated using the XOR operation. The circular shift-based matching is performed from which the minimum matching score is 
considered after several circular shifts. Alternatively, Li Ma proposed the orthogonal moment based method [23] wherein the Gauss-Hermite moments of 1D signals are used as distinguishing features. These moments are effective to characterize the local details of the signal. Ten intensity signals were generated and four different order (1-4) moments were used. The feature vector was constructed by concatenating these features. The nearest center classifier based on cosine similarity measure was adopted for classification in a low dimensional feature space. The method by Li Ma was further improved by Zhenan Sun [24] where in the local feature based classifier was combined with an iris blob matcher. The blob matching aimed at finding the spatial correspondences between the blocks in the input image and that in the stored model. The similarity is based on the number of matched block pairs. The block attributes are recorded as centroid coordinates, area and second order central moments.

The method by Jong Gook Ko [25] is based on cumulative-sumbased change points. The iris segmentation uses Daugman's method and the segmented image is normalized to $64 \times 300$ pixel area. The feature extraction is performed using cumulative sums on groups of basic cells where each cell is of size $3 \times 10$. An average gray value represents the cell region for calculation. The cell regions are grouped horizontally and vertically and cumulative sums are calculated over each group. The iris feature codes are generated based on the sum in both horizontal and vertical directions. The maximum and minimum of the sum is calculated. For the summation values that lie between these two values, if the sum is on upward slope the cell's iriscode is set to 1. When the sum is on the downward slope, the cell's iriscode is set to 2 otherwise cell's iris code is set to zero. Matching is performed using HD. A region-based feature extraction method based on 2D-Discrete Wavelet Transform was proposed by N.Tajbakhsh [26]. The iris texture is partitioned into 32x32 pixel blocks and then the 2D wavelet decomposition is performed on every block. The Gauss-Laguerre filter is used to generate a binary matrix similar to iris code in Daugman method.

\subsection{Approach using Independent Component Analysis}

The iris recognition system developed by Ya-Ping Huang [27] adopts Independent Component Analysis (ICA) to extract iris texture features. Image acquisition is performed at different illumination and noise levels. The iris localization is performed using integrodifferential operator and parabolic curve fitting. From the inner to outer boundary of iris, fixed number of concentric circles $\mathrm{n}$ with $\mathrm{m}$ samples on each circle is obtained. This is represented as a matrix $\mathrm{n} \times \mathrm{m}$ for a specific iris image which is invariant to rotation and size. The independent components are uncorrelated, determined from the feature coefficients. The feature coefficients are non-Gaussian and mutually independent. The basis function used is kurtosis. The independent components are estimated and encoded. The centre of each class is determined by competitive learning mechanism which is stored as the iriscode for a person. The average Euclidean distance classifier is used to recognize iris patterns.

\subsection{Iris authentication based on Continuous Dynamic Programming}

The technique proposed by Radhika [28] authenticates iris based on kinematic characteristics, acceleration. Pupil extraction begins by identifying the highest peak from the histogram which provides the threshold for lower intensity values of the eye image. All the connected components in sample eye image less than threshold intensity value are labeled. By selecting the maximum area component we arrive at pupil area of the eye. Normalised bounding rectangle is implemented using centre of pupil to crop iris. Continuous dynamic programming is used with the concept of comparing shape characteristics part wise. The acceleration plot is segmented and parts of acceleration curve are used to verify with input's acceleration curve. For iris samples, rate of change of gray level intensities within bounding box forms acceleration feature plot. The implementation is based the concept of accumulated minimum local distances between a reference template and input sample. The reference template is obtained using leave one out method. The distance measure is the count of directional changes in acceleration plot. The local distances are directional changes in respective segmented slots of the acceleration plot. The methods are summarized in Table 2.

Table 2. Iris Recognition Methodologies

\begin{tabular}{|c|c|c|}
\hline Group & Size of Database & Results \\
\hline Daugman & 4258 images & EER: $0.08 \%$. \\
\hline Wildes et al. & 60 images & EER: $1.76 \%$ \\
\hline $\begin{array}{l}\text { Boles and } \\
\text { Boashash }\end{array}$ & Real images & EER: $8.13 \%$ \\
\hline Li Ma & $\begin{array}{l}2245 \text { images } \\
\text { (CASIA) }\end{array}$ & $\begin{array}{l}\text { Correct Recognition } \\
\text { Rate: } 94.33 \% .\end{array}$ \\
\hline Masek & 624 images & $\begin{array}{l}\text { FAR: } 0.005 \% \\
\text { FRR: } 0.238 \% \text {. }\end{array}$ \\
\hline Ya-Ping Huang & Real images & $\begin{array}{l}81.3 \% \text { for blurred iris, } \\
93.8 \% \text { for variant } \\
\text { illumination and } \\
62.5 \% \text { for noise } \\
\text { interference images. }\end{array}$ \\
\hline $\begin{array}{l}\text { H. Proenca and } \\
\text { L.A.Alexandre }\end{array}$ & $\begin{array}{l}1877 \text { images } \\
\text { (UBIRIS) }\end{array}$ & $\begin{array}{l}\text { Recognition rate: } 98.02 \% \\
\text { (session 1), } \\
97.88 \%(\text { session } 2)\end{array}$ \\
\hline Tisse and Martin & 300 images & FRR: $8 \%$ \\
\hline Xiaomei Liu & $\begin{array}{l}12000 \text { images } \\
\text { (ICE) }\end{array}$ & Recognition rate: $96.61 \%$ \\
\hline Jinyo Zuo & $\begin{array}{l}450 \text { images } \\
\text { (MMU1) }\end{array}$ & EER: $0.0256 \%$ \\
\hline
\end{tabular}




\begin{tabular}{|c|c|c|}
\hline $\begin{array}{l}\text { Jong Gook Ko et } \\
\text { al }\end{array}$ & $\begin{array}{l}\text { (i) } 820 \text { images } \\
\text { from } 82 \\
\text { individuals } \\
\text { (ii) } 756 \text { images } \\
\text { (CASIA) }\end{array}$ & $\begin{array}{l}\text { FAR: } 5.26 \% \text {, } \\
\text { FRR: } 0.25 \% \\
\text { Recognition rate: } 98.21 \%\end{array}$ \\
\hline N.Tajbakhsh & $\begin{array}{l}1877 \text { images } \\
\text { (UBIRIS) }\end{array}$ & $\begin{array}{l}0.66 \% \text { ERR, } 4.10 \% \text { FRR } \\
\text { and } 0.01 \% \text { FAR. }\end{array}$ \\
\hline $\begin{array}{l}\text { Karen } \\
\text { Hollingsworth }\end{array}$ & $\begin{array}{l}\text { (a) } 1226 \text { images } \\
\text { from } 24 \text { subjects } \\
\text { (ICE). } \\
\text { (b) } 1061 \text { videos } \\
\text { from } 296 \text { eyes. } \\
\text { (c) ICE database } \\
\text { (d) } 1263 \text { images } \\
\text { from } 18 \text { subjects } \\
\text { (ICE). }\end{array}$ & $\begin{array}{l}\text { (a) } \mathrm{HD}=7.48 \\
\text { (b) } \mathrm{EER}=3.88 \times 10^{-3}, \\
\mathrm{FRR}=7.61 \times 10^{-6}, \\
\mathrm{FAR}=0.001 \\
\text { (c) } \mathrm{HD}=0.15 \\
\text { (d) } \mathrm{FRR}=0.271, \\
\mathrm{FAR}=0.001, \mathrm{EER}=0.068 \\
\text { for large pupil subset }\end{array}$ \\
\hline Radhika et al & $\begin{array}{l}\text { (a) } 1205 \text { images } \\
\text { (UBIRIS) } \\
\text { (b) } 1200 \text { images } \\
\text { (CASIAv2) }\end{array}$ & $\begin{array}{l}\text { Acceptance Rate: } 98 \% \\
\text { Rejection Rate } 97 \%\end{array}$ \\
\hline
\end{tabular}

\section{PRODUCTS AND SOLUTIONS}

Iris authentication products are used in significant applications like civilian identification management programs. Some of the products are as follows. The iris recognition system by L-1 Identity Solutions is based on Daugman method. The algorithm has been used in the National Institute of Standards and Technology Iris Exchange [NIST IREX] testing program. The Offender Identification System [Offender-ID] supports identification of prisoners in jail environment. PIER 2.4 provides mobile identification with iris technology in a real time environment. The Handheld Interagency Identity Detection Equipment [HIIDE] is a multi-biometric handheld device. It is used in defense agencies and in remote or centralized enrolments. The LG IrisAccess, Panasonic BM-ET200, Oki, IBM, IrisGuard IG-AD100, Sagem, Securimetrics and Argus systems work by analyzing the iris patterns and converting them into digital templates.

\section{CONCLUSION}

The physiological characteristics are relatively unique to an individual. An approach to reliable visual recognition of persons is achieved by iris patterns. The other approaches are based on discrete cosine transforms, corner detection and parametric template methods. The future work in real applications utilization to support generation of compact iris codes for mobile phones and PDAs. In this paper, an attempt has been made to present an insight of different iris recognition methods. The survey of the techniques provides a platform for the development of the novel techniques in this area as future work.

\section{REFERENCES}

[1] A. Bertillon. La couleur de Piris. Revue scientifique, Vol. 36, No. 3, pp. 65-73, 1885.

[2] Leonard Flom and Aran Safir, "Iris recognition system", U.S.Patent 1244552, 1987.
[3] R. Johnston, "Can iris patterns be used to identify people?", Los Alamos National Laboratory, Chemical and Laser Sciences Division Annual Report LA-12331-PR, pp. 81-86, 1992.

[4] Kevin W. Bowyer, Karen Hollingsworth, Patrick J. Flynn, "Image Understanding for Iris Biometrics: A Survey, Computer Vision and Image Understanding", Vol. 110, Issue 2, pp. 281307, 2008.

[5] W. Ketchantang, S. Derrode, S. Bourennane and L. Martin, "Video Pupil Tracking for Iris Based Identification", Advanced Concepts for Intelligent Vision Systems, LNCS 3708, pp. 1-8, 2005 .

[6] Zhaofeng He, Tieniu Tan and Zhenan Sun, "Iris Localization via Pulling and Pushing", International Conference on Pattern Recognition, pp. 366 - 369, 2006.

[7] Mira J. and Mayer J., "Image feature extraction for application of biometric identification of iris: a morphological approach", IEEE Proc. XVI Brazilian Symposium on Computer Graphics and Image Processing, pp. 391- 398, 2003.

[8] Guodong Guo, Micheal J. Jones, "Iris extraction based on Intensity Gradient and Texture Difference", IEEE Workshop on Applications of Computer Vision, pp. 1-6, 2008.

[9] Proenca H, and Alexandre, L.A, "UBIRIS: a noisy iris image database", ICIAP 2005, International Conference on Image Analysis and Processing, pp. 970-977, 2005.

[10] Li Ma, Tieniu Tan, Yunhong Wang, Dexin Zhang, "Personal Identification based on Iris Texture Analysis", IEEE Transactions on Pattern Analysis and Machine Intelligence, Vol.25, No.12, pp. 1519 - 1533, 2003.

[11] Daugman J, "How iris recognition works", IEEE Transactions CSV, Vol.14, No.1, pp. 21-30, 2004.

[12] J. Daugman, "High Confidence Visual Recognition by a test of Statistical Independence", IEEE Trans. Pattern Analysis and Machine Intelligence, Vol. 15, No.11, pp.1148-1161,1993.

[13] Xianchao Qui, Zhenan Sun and Tieniu Tan, "Learning Appearance primitives of iris images for ethnic classification", International Conference on Image Processing, pp. 405-408, 2007.

[14] Martin-Roche D., Sanchez-Avila C. and Sanchez-Reillo R., "Iris recognition for biometric identification using dyadic wavelet transform zero-crossing", IEEE Aerosp. Electron. Syst. Mag., Vol. 17, Issue 10, pp. 3-6, 2002

[15] Libor Masek, "Recognition of Human Iris Patterns for Biometric Identification", School of Computer Science and Soft Engineering, The University of Western Australia, 2003.

[16] Xiaomei Liu, Bowyer K.W., Flynn, P.J., "Experimental Evaluation of Iris Recognition", IEEE Computer Society Conference on Computer Vision and Pattern Recognition Workshops, pp. 158-165, 2005.

[17] Karen Hollingsworth, Sarah Baker, Sarah Ring, Kevin W. Bowyer and Patrick J. Flynn, "Recent research results in iris biometrics", Proceedings of the SPIE, Vol. 7306, pp. 73061Y73061Y-10, 2009. 
[18] R. Wildes, J. Asmuth, G. Green, S. Hsu, R. Kolczynski, J. Matey, and S. McBride, "A machine-vision system for iris recognition”, Machine Visual Application, Vol. 9, pp. 1-8, 1996.

[19] R. Wildes, "Iris recognition: an emerging biometric technology", IEEE Proceedings, Vol. 85, pp. 1348-1363, 1997.

[20] R.P.Wildes, J.C.Asmuth, G.L. Green, S.C.Hsu, R.J,Kolczynski, J.R.Matey, S.E.McBride, David Sarno_ Res. Center, Princeton, NJ, "A System for Automated Iris Recognition", Proceedings of the Second IEEE Workshop on Applications of Computer Vision, 1994.

[21] Emine Krichen, M.Anouar Mellakh, Sonia Garcia-Salicetti, Bernadette Dorizzi, "Iris identification using Wavelet packets", International Conference on Pattern Recognition, Vol. 4, pp. 33538, 2004.

[22] W. W. Boles and B. Boashash , "A Human Identification Technique Using Images of the Iris and Wavelet Transform", IEEE Transactions On Signal Processing, Vol. 46, No. 4, April 1998.

[23] Li Ma, Tieniu Tan, Yunhong Wang, Dexin Zhang, "Efficient Iris Recognition by characterizing Key Local Variations", IEEE Transactions on Image Processing, Vol. 13, No.6, 2004.
[24] Zhenan Sun, Yunhong Wang, Tieniu Tan and Jiali Cui, "Improving Iris Recognition Accuracy Via Cascaded Classifiers", IEEE Transactions on Systems, Man and Cybernetics, Part C, Vol. 35, No. 3, pp. 435-441, 2005.

[25] Jong Gook Ko, Youn Hee Gil, Jang Hee Yoo, Kyo Il Chung, "Method of iris recognition using cumulative-sum-based change point analysis and apparatus using the same", US Patent 20070014438, 2007.

[26] Nima Tajbakhsh, Khashayar Misaghian and Naghmeh Mohammadi Bandari, "A Region-Based iris feature extraction method based on 2D-wavelet transform", Bio_ID_MultiComm2009, LNCS 5707, pp. 301-307, 2009.

[27] Ya-Ping Huang, Si-Wei Luo, En- Yi Chen, “An efficient iris recognition system", International Conference on Machine Learning and Cybernetics, pp. 450-454, 2002.

[28] K.R.Radhika, S.V.Sheela, M.K.Venkatesha and G.N.Sekhar, "Multimodal Authentication using Continuous Dynamic Programming", International Conference on Biometric ID Management and Multimodal Communication, Springer LNCS 5707, 228-235, 2009.

[29] CASIA-IrisV3, http://www.cbsr.ia.ac.cn/IrisDatabase. htm 